\title{
High Acute Myeloid Leukemia derived VEGFA levels are associated with a specific vascular morphology in the leukemic bone marrow
}

\author{
Alida C. Weidenaar • Arja ter Elst • Gineke Koopmans-Klein • Stefano Rosati • \\ Wilfred F. A. den Dunnen • Tiny Meeuwsen-de Boer - Willem A. Kamps • \\ Edo Vellenga • Eveline S. J. M. de Bont
}

Accepted: 9 February 2011 / Published online: 6 April 2011

(C) The Author(s) 2011. This article is published with open access at Springerlink.com

\begin{abstract}
Background Acute Myeloid Leukemia (AML) bone marrow biopsies at diagnosis display enhanced angiogenesis and increased VEGFA expression. In a xenograft mouse model it was described that availability of free VEGFA versus bound VEGFA is related to different vascular morphology. In this study we investigate the relationship between vascular morphology within AML bone marrow biopsies and AML derived VEGFA levels.

Methods Vessel count and surface area (Chalkley count) were calculated in AML bone marrow biopsies at diagnosis $(n=32)$, at remission $(n=8)$ and Normal Bone Marrow $(n=32)$ using immunohistochemical staining for FVIII, CD31, CTIV, SMA and VEGFA. VEGFA protein levels were measured.

Results High vessel count was associated with an immature vessel status. Combining vessel count and Chalkley count
\end{abstract}

This work was supported by a grant from the 'Dutch Cancer Society' to E.S.J.M. de Bont (3661).

A. C. Weidenaar · A. ter Elst $\cdot$ G. Koopmans-Klein ·

T. Meeuwsen-de Boer · W. A. Kamps • E. S. J. M. de Bont $(\bowtie)$

Division of Pediatric Oncology/Hematology, Department of

Pediatrics, Beatrix Children's Hospital, University Medical Center

Groningen, University of Groningen,

P.O.Box 30.001, 9700 RB Groningen, the Netherlands

e-mail: e.s.j.m.de.bont@bkk.umcg.nl

S. Rosati - W. F. A. den Dunnen

Division of Pathology, Department of Pathology and Medical

Biology, University Medical Center Groningen, University of Groningen,

Groningen, the Netherlands

E. Vellenga

Division of Hematology, Department of Medicine, University

Medical Center Groningen, University of Groningen,

Groningen, the Netherlands different vessel morphology patterns were quantified within AML bone marrow biopsies. Three different subgroups could be distinguished. The subgroup $37.5 \%$ of the samples) exhibiting a high vessel count and vessels with predominantly large lumen (normal Chalkley count) was associated with high secreted VEGFA protein levels.

Conclusion Different vasculature patterns are seen in AML bone marrow biopsies, defined by combining number and size of vessel. These quantified morphology patterns, combined with VEGFA levels, might be of value in the success of VEGF/VEGFR-signaling interference approaches.

Keywords Acute Myeloid Leukemia - VEGFA .

Angiogenesis · Vessel morphology

\section{Introduction}

Acute Myeloid Leukemia (AML) is a disease with a poor outcome; the overall survival at 5 years is moderate to poor depending on age and cytogenetics [3, 6, 12]. At diagnosis an enhanced microvessel density (MVD) in bone marrow biopsies has been observed, which is restored to normal levels when a complete remission has been achieved [5, 21]. In addition, in AML patients a high bone marrow blood flow and high tissue perfusion measured by MRI techniques is related to a significantly decreased diseasefree survival and overall survival [14]. The enhanced bone marrow vascularization is correlated with an increased expression of Vascular Endothelial Growth Factor (VEGF) A [5]. Moreover, the level of VEGFA is an independent prognostic factor for treatment outcome in AML [1, 5]. Clinical trials targeting the VEGF/VEGFR signaling path- 
way in patients with relapsed or refractory AML are reported and showed beneficial effects in a subset of patients. Treatment with Bevacizumab, a monoclonal antibody against VEGFA, following chemotherapy achieved a complete remission in $33 \%$ of patients with refractory AML [18]. A VEGF receptor tyrosine kinase inhibitor, SU5416, showed a significantly higher response rate and reduction of bone marrow microvessel density in patients with AML blasts expressing high levels of VEGF mRNA than in patients with low VEGF expression [9]. These reports show that there is at least heterogeneity in treatment response related to VEGFA levels.

Normal vasculature is highly organized and consists of vessels formed by Endothelial Cells (ECs) which are surrounded by basal membrane components and pericytes to provide structural and functional support [4]. This mature vasculature pattern is thought to be the result of a coordinated balance between pro-angiogenic and antiangiogenic factors (reviewed in $[10,11]$ ). In contrast to the formation of normal blood vasculature, xenograft animal models showed that tumor vasculature is structurally and functionally abnormal; the hierarchical structure has been lost and dilated vessels and/or angiogenic vessel sprouts appeared $[15,23,25]$. The process of vessel formation comprises a disturbance in the balance of angiogenic factors, where VEGFA seems to play an important role [8]. These kind of differences in tumor vessel morphology seemed to be related to the availability of VEGFA; bound VEGFA results in angiogenic sprouting, whereas free or cleaved VEGFA results in dilated vessels in a xenograft mouse model [20].

In the present study we identified for the first time different structured networks of vessels in the bone marrow of newly diagnosed AML patients. Three different subgroups could be distinguished. In addition, one of the subgroups containing a high vessel count and a high vessel surface area was associated with high VEGFA protein level secreted by AML cells.

\section{Materials and methods}

\subsection{Leukemic cells and bone marrow biopsy specimens}

Bone marrow biopsies and leukemic cells of 32 newly diagnosed AML-patients were obtained at presentation after informed consent in accordance with the regulations and protocols sanctioned by the medical ethical committee. Clinical and cell biological data, including (cyto-)genetics as well as treatment outcome, were obtained from UMCG patient database. Table 1 summarizes patient characteristics of the AML patients. AML patients were treated following Dutch Hemato-Oncology Group (HOVON) protocols. In 8 AML patients a bone marrow biopsy was available at the time of complete remission. Normal control bone marrow biopsies were obtained from patients who underwent bone marrow biopsies as a staging procedure for a solid tumor or hematological insufficiency (e.g. anemia, leucopenia) and were diagnosed as normal $(n=32)$. Mononuclear cells (MNCs) were separated using Lymphoprep (Nycomed, Oslo, Norway) density gradients and cryopreserved in liquid nitrogen until use. Cryopreserved AML cells were thawed rapidly at $37^{\circ} \mathrm{C}$ diluted in a $5 \times$ volume of normal calf serum (NCS) as previously described [7]. The remaining pellet was T cell-depleted by sheep red blood cells and separated over a Lymphoprep density gradient. The remaining blast cell population contained more than 95\% AML cells and is hereafter referred to as AML cells.

\subsection{Culture conditions for leukemic cells}

AML cells were cultured at $1 \times 10^{6}$ cells $/ \mathrm{ml}$ in $\mathrm{X}$-vivo 10 medium supplemented with penicillin/streptomycin for $24 \mathrm{~h}$ (FCS, Hyclone, Logan, UT, USA). Supernatants were harvested and centrifuged.

\subsection{Detection of proteins using enzyme-linked immunosorbent assay (ELISA)}

Secretion of human VEGFA by AML cells was detected in the supernatant using commercially available ELISAs (Quantikine immunoassays, R \& D, Minneapolis, MN, USA) following the manufacturer's instructions. VEGFA levels were detectable in 11 of the 30 samples.

\subsection{RNA extraction and quantitative RT-PCR}

Total RNA of the AML cells was extracted with the RNeasy mini kit according to the manufacturer's description (Qiagen, Breda, the Netherlands). An on column DNAse digestion was performed to purify the obtained RNA. cDNA was prepared by reverse transcription as described by the manufacturer (MBI Fermentas GmbH, St. Leon-Rot, Germany). The final reaction mix had a final volume of $20 \mu \mathrm{l}$ and contained $1 \mu \mathrm{g}$ total RNA, $1 \mu \mathrm{g}$ random hexamers, $1 \mathrm{mM}$ of dNTPs, $20 \mathrm{U}$ RNAse inhibitor and $200 \mathrm{U}$ of RevertAid ${ }^{\mathrm{TM}}$ M-MuLV Reverse Transcriptase. Quantitative LightCycler PCR was performed using the LightCycler-FastStart DNA Master SYBR Green I system using $1 \mu \mathrm{l}$ of a 10-fold diluted cDNA in each PCR reaction in a final volume of $10 \mu \mathrm{l}$ (Roche Molecular Biochemicals, Mannheim, Germany). Specific primers for $\beta_{2}$-microglobulin were sense (CCA GCA GAG AAT GGA AAG TC) and antisense (GAT GCT TAC ATG TCT CG), PCR product $268 \mathrm{bp}, 22$ cycles, $55^{\circ} \mathrm{C}$; for VEGFA sense (GAG TGT GTG CCC ACT GAG GAG TCC AAC) and antisense (CTC CTG CCC GGC TCA CCG CCT CGG 
Table 1 Patient characteristics

Characteristics-age, WBC, percentage peripheral blasts and platelets - and staining counts are given as median (range)

\begin{tabular}{|c|c|c|c|}
\hline Characteristic & AML patients & Normal bone marrow & AML remission \\
\hline No. & 32 & 32 & 8 \\
\hline Age at diagnosis, $\mathrm{y}$ & $54(20-73)$ & $58(17-83)$ & $48(20-64)$ \\
\hline Sex, male/female & $17 / 15$ & $17 / 15$ & $4 / 4$ \\
\hline White blood cell count $\left(\mathrm{x} 10^{9} / \mathrm{L}\right)$ & $28(8-269)$ & & \\
\hline Peripheral blasts (\%) & $62(3-97)$ & & \\
\hline Platelets (x $\left.10^{9} / \mathrm{L}\right)$ & $48.7(1.1-241.0)$ & & \\
\hline \multicolumn{4}{|l|}{ Cytogenetics, n (\%) } \\
\hline Favorable & $2(6)$ & & \\
\hline Intermediate & $18(56)$ & & \\
\hline Unfavorable & $4(13)$ & & \\
\hline Not available & $8(25)$ & & \\
\hline \multicolumn{4}{|l|}{ SCT } \\
\hline No SCT & 22 & & \\
\hline Allogeneic SCT & 7 & & \\
\hline Autologous SCT & 3 & & \\
\hline \multicolumn{4}{|l|}{ Cycles to CR, n (\%) } \\
\hline 1 & $13(41)$ & & \\
\hline 2 & $9(28)$ & & \\
\hline 3 & $2(6)$ & & \\
\hline No CR & $8(25)$ & & \\
\hline Relapse, n (\%) & $18(56)$ & & \\
\hline Dead/alive & $24 / 8$ & & \\
\hline \multicolumn{4}{|l|}{ Staining } \\
\hline Vessel count, n (\%) & $32(100)$ & $32(100)$ & $8(100)$ \\
\hline counts & $16.2(5.8-33.8)$ & $7.4(2.0-15.0)$ & $5.7(1.0-9.8)$ \\
\hline Chalkley count, n (\%) & $32(100)$ & $32(100)$ & $8(100)$ \\
\hline counts & $5.4(3.2-11.0)$ & $5.3(3.3-10.3)$ & $7.5(6.0-10.0)$ \\
\hline \%CTIV-positive vessels, n (\%) & $29(91)$ & $28(88)$ & $7(88)$ \\
\hline counts & $87.3(2.0-100.0)$ & $100.0(0.0-100.0)$ & $61.2(0.0-100.0)$ \\
\hline \%SMA-positive vessels, n (\%) & $26(81)$ & $31(97)$ & $8(100)$ \\
\hline counts & $35.0(8.9-100.0)$ & $73.1(22.7-100.0)$ & $54.9(18.4-100.0)$ \\
\hline VEGFA, n (\%) & $32(100)$ & $13(41)$ & $8(100)$ \\
\hline
\end{tabular}

CTT), PCR product 177, 312, 384 bp, 32 cycles, $60^{\circ} \mathrm{C}$. PCR products were subjected to melting curve analysis using the Light Cycler system to exclude the amplification of unspecific products. Finally, the PCR products were analyzed by conventional agarose gel electrophoresis. The expression of VEGF was standardized for expression of $\beta_{2^{-}}$ microglobulin (Arbitrary Units, AU). Serial cDNA dilutions of a mixture of all patient samples were used to generate standard curves. The expression of each gene in each sample was analyzed in duplicate. The regression coefficients of the standard curves were $\geq 0.99$.

\subsection{Immunohistochemical analysis}

Bone marrow paraffin biopsy specimens were cut into 4$\mu \mathrm{m}$ sections and stained for Factor VIII related antigen (FVIIIRA, also known as von Willebrand Factor), Collagen type IV (CTIV), CD31, SMA and VEGFA. Sections were deparaffined, blocked for endogenous peroxidase with $0.3 \% \mathrm{H}_{2} \mathrm{O}_{2}$, blocked for antigen retrieval with $1 \%$ protease, immunostained for FVIIIRA (DAKO, AS, Glostrup, Denmark) and amplified with biotin-streptavidin HRPO (DAKO). The sections stained with CD31 (DAKO), SMA (DAKO) and CTIV (Southern Biotech, Birmingham, Alabama, USA) were treated the same way except for the antigen retrieval with $0.1 \mathrm{M}$ Tris- $\mathrm{HCl} \mathrm{pH} 9.0$ at $80^{\circ} \mathrm{C}$ over night (SMA) or for $30 \mathrm{~min}$ in the microwave (CD31, CTIV). VEGFA (Santa Cruz, Heidelberg, Germany) stainings were blocked with $0.1 \mathrm{M}$ Tris- $\mathrm{HCl} \mathrm{pH} 9.0$ for $15 \mathrm{~min}$ in the microwave and treated with an avidine/biotine block. The color reaction was assessed by adding substrate for peroxidase. After that the slides are colored with hematoxillin. Negative controls were produced by using nonspecific IgG as the primary antibody (DAKO). 
The vessel count measured by FVIIIRA was assessed using light microscopy in areas of the slide containing the highest numbers of blood vessels (hotspots). After the hotspots were identified, the total number of vessels per selected image was counted at $\times 400$ magnification. At least five fields were counted for each section, and the true vessel number was expressed as the mean of five counts. Quantification of the relative area estimate of the vessels was assessed using the Chalkley point overlap morphometric technique as described before $[22,24]$. This method entails the use of an ocular grid with 25 random points. The ocular grid was turned to maximize the overlap between points on the grid and the vessels for five hotspots at $400 \times$ magnification. The number of overlapping points was counted for each of the hot spots, and the Chalkley count was expressed as mean value of the five counts. The Chalkley count was determined on FVIIIRA, CD31 and CTIV staining with a correlation coefficient of rho $=0.714$, $p=0.001$. Biopsies of 6 AML-patients and 1 normal bone marrow control could not be evaluated for SMA. Pericyte coverage (\%SMA-positive vessels) and basal membrane coverage (\%CTIV-positive vessels) were expressed as the percentage of FVIII positive vessels. The intensity of the VEGFA staining was analyzed semiquantitatively: $0=$ no staining, $1=$ slight staining, $2=$ moderate staining, $3=$ maximal staining. All stainings were evaluated by at least two separate investigators who had no knowledge of patient characteristics. Data was summarized in Table 1.

\subsection{Statistical analysis}

Statistical analysis was performed with PASW Statistics 18. The significance of changes in vessel counts, \% SMA-positive vessels, VEGFA production and patient characteristics between three defined morphology groups or cytogenetic groups was assessed using non-parametric tests (Kruskal Wallis Test). The \% SMA-positive vessels between two groups, and the correlation between vessel count and sex were determined with the non-parametric Mann Whitney- $U$ test. Correlation between vessel count and patient characteristics was measured using the Spearman's rho. Correlation between vessel count and Chalkley count for the AML biopsies at diagnosis was measured using the Spearman's rho. A significant difference was defined as a $p$-value $\leq 0.05$.

\section{Results}

3.1 Immature vessel status in AML bone marrow biopsies at diagnosis

Vessel count was significantly $(p<0.001)$ increased in AML bone marrow at diagnosis (median: 16.2 microvessels/hpf, range 5.8-33.8, $n=32$ ) compared with normal control bone marrow (NBM, median: 7.4 microvessels/hpf, range 2.0 $15.0, n=32$ ), and returned to normal levels when complete remission was achieved (median: 5.7 microvessels/hpf, range $1.0-9.8, n=8$ ) underscoring previous studies (Fig. 1a) $[5$, 21]. Based on the fact that $>95 \%$ of the Normal Bone Marrow biopsies and AML remission biopsies had a vessel count below 13 microvessels/hpf, it appeared that a higher vessel count was aberrant. Therefore, the value of 13 microvessels/hpf was taken as cut-off point for categoriza-
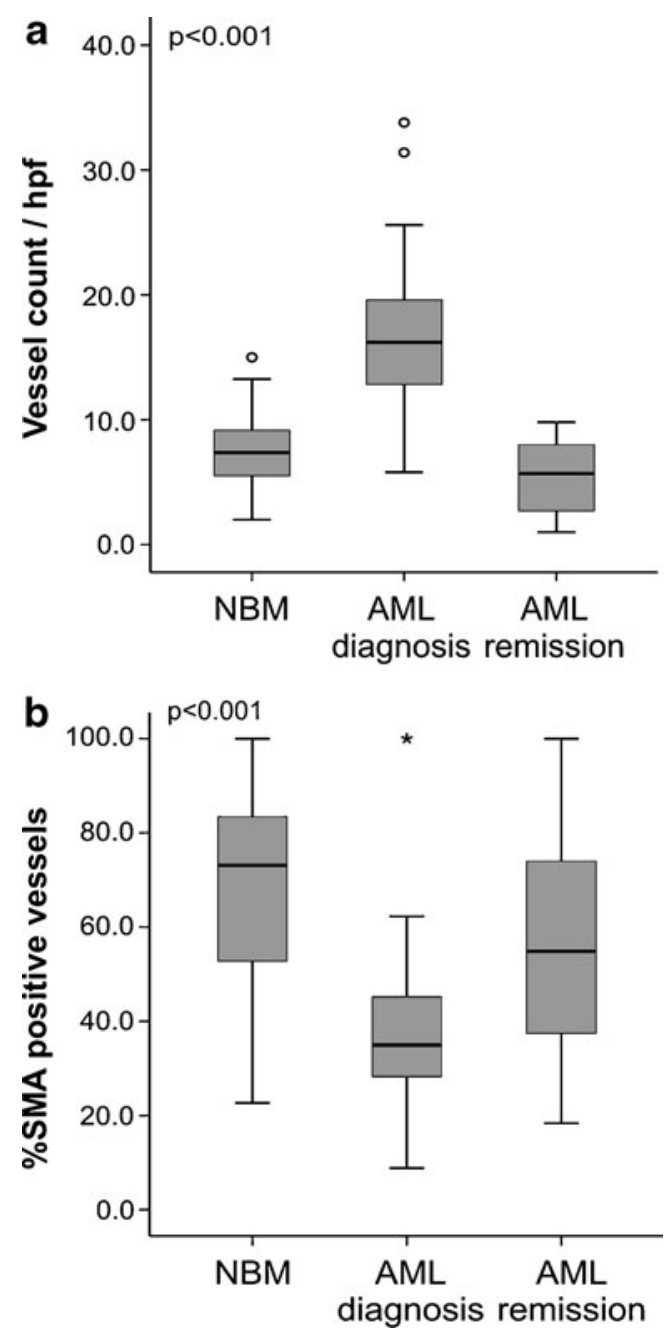

Fig. 1 Bone marrow vessel count and pericyte coverage in AML (a) Boxplot of vessel count in AML bone marrow compared with normal bone marrow and AML remission. Vessel count in bone marrow biopsies of AML patients is significantly $(p<0.001)$ higher than in NBM or AML remission group. Median values are represented by a bar in the boxplot. (b) Boxplot of percentage SMA-positive vessels in bone marrow biopsies of newly diagnosed AML patients, normal controls and AML patients in remission. Pericyte coverage is significantly $(p<0.001)$ lower in bone marrow biopsies of AML patients at diagnosis. Median values are represented by a bar in the boxplot 
tion into 'low vessel count' (13 or less) and 'high vessel count' (more than 13). Of the AML bone marrow biopsies $75 \%$ (24/32) were categorized as biopsies with a 'high vessel count'. No significant correlation was found between vessel count and patient characteristics such as WBC count $(p=$ 0.65 , Spearman's rho: -0.08 , $)$, platelets $(p=0.85$, Spearman's rho: 0.04$)$, blast percentage at diagnosis $(p=0.27$, Spearman's rho: -0.16$)$, and age ( $p=0.67$, Spearman's rho: 0.08$)$, and no significant difference in vessel count was found for cytogenetics ( $p=0.27$, Kruskal-Wallis test) and sex $(p=0.58$, Mann Whitney $U$ test).

Basal membrane surrounding of the vessels was determined by the percentage collagen type IV (\%CTIV) positive vessels. Median value in the AML bone marrow biopsies was $87.3 \%$ (range $2.1-100.0 \%, n=29$ ) compared with $100.0 \%$ in the NBM (range $0.0-100.0 \%, n=28$ ) and $61.2 \%$ in the AML remission bone marrow biopsies (range $0.0-100.0 \%, n=7)$. No significant difference was found between the groups, suggesting that the number of endothelial cell structures surrounded by basal membranes is comparable. Since vessel stabilization requires pericyte coverage of the vascular sprouts, we studied the number of vessels positive for Smooth Muscle Actin (SMA), a marker for pericytes. In AML bone marrow biopsies at diagnosis $35.0 \%$ of vessels were pericyte-coated (range $8.9-100.0 \%$, $n=26$ ) versus $73.1 \%$ of vessels in the NBM (range $22.7-$ $100.0 \%, n=31$ ) and $54.9 \%$ in the AML remission group (range 18.4-100.0\%, $n=8)(p<0.001$, Fig. 1b). Interestingly, the percentage vessels coated with pericytes was significantly $(p=0.04)$ higher in the 'low vessel count' group (45\%, range $23-100 \%, n=7)$ compared with the biopsies with 'high vessel count' (29\%, range $9-57 \%, n=$ 19), indicating that high vessel count in AML bone marrow is related to a more immature vessel status.

3.2 Different vasculature morphology patterns within AML bone marrow biopsies at diagnosis

Assessing vessel morphology it appeared that different vasculature patterns within the AML bone marrow biopsies were seen (Fig. 2); biopsies displaying a high number of vessels with predominantly a large lumen and thin walls, but also biopsies exhibiting a high vessel count with mainly a network of small vessels with thin walls, narrow lumen and branching. To quantify this observation the vessel count was combined with a method to measure the vessel surface area, the Chalkley count; this is a quantification technique which determines a number of grid points that hit stained vessels or lumina. Figure 3 shows the results for vessel count and Chalkley count for the AML biopsies at diagnosis (median Chalkley count: 5.4, range 3.2-11.0), Normal Bone Marrow samples (median Chalkley count: 5.3, range 3.3-10.3) and $\mathrm{AML}$ at remission (median
Chalkley count: 7.5, range 6.0-10.0). Within the group displaying a 'high vessel count' we further categorized the biopsies into two subgroups. The median value of 5.4 was taken as a cut-off for categorization into 'low' (5.4 or less) and 'high' (more than 5.4) Chalkley count. The first subgroup $(12 / 32,37.5 \%)$ had a high Chalkley count (group I in Fig. 3) and was defined as'vessel hyperplasia', characterized by vessels with a predominantly large lumen and thin walls. The second subgroup (12/32, 37.5\%) had a low Chalkley count (group II in Fig. 3) and was defined as 'angiogenic sprouting', displaying a network of small vessels with thin walls, narrow lumen and branching. Regarding patient characteristics the number of platelets at diagnosis was significantly $(p=0.02)$ higher in the 'vessel hyperplasia' patients compared with the other two defined morphology patterns (data not shown). No differences were found between the morphological identified subgroups and other patient characteristics.

3.3 High free VEGFA levels excreted by AML cells related to vessel hyperplasia morphology

In a xenograft mouse model the availability of free versus bound VEGFA was related to the appearance of the tumor vasculature [20]. Since AML cells can secrete VEGFA we hypothesized that the observed differences in AML bone marrow vessel morphology could be related to excreted VEGFA protein levels. Immunohistochemical staining for total VEGFA protein expression showed a diffuse VEGFA distribution in all AML biopsies at diagnosis: the cytoplasm of leukemic blasts as well as the intercellular areas were mainly positive for VEGFA (Fig. 2). In contrast, Normal Bone Marrow biopsies showed a different distribution pattern, similar to biopsies of patients in complete remission: highly positive myeloid cells, a positive cytoplasm of megakaryocytes and erythroid cells that were negative for VEGFA (not shown). Immunohistochemistry (IHC) of the AML biopsies at diagnosis showed that the total amount of protein VEGFA was comparable in all samples. In accordance with the IHC the VEGFA mRNA level was not significantly $(p=0.24)$ different within the three defined subgroups ('low vessel count' 3.1 AU, range 2.0-13.1; 'vessel hyperplasia' 6.1 AU, range 2.0-49.3; 'angiogenic sprouting' median 15.1 AU, range 2.6-64.6). In contrast, AML derived excreted VEGFA protein levels in AML at diagnosis were significantly $(p=0.007)$ higher in the 'vessel hyperplasia' morphology subgroup compared to the 'angiogenic sprouting' subgroup and the 'low vessel count' biopsies (Fig. 4) (median $2.00 \mathrm{pg} / \mathrm{ml}$, range 2.00-47.77, $n=$ 30). Overall, we conclude that the total amount of VEGFA protein level and mRNA is similar in the AML biopsies at diagnosis whereas AML derived VEGFA levels are higher in the group displaying a'vessel hyperplasia' pattern. 
Fig. 2 Morphology patterns in AML bone marrow biopsies. Dilated vessels in panel $\mathbf{a}$ and sprouting vessels in panel $\mathbf{b}$. Representative pictures of bone marrow immunohistochemistry for FVIII, CTIV and VEGFA $(\times 400)$

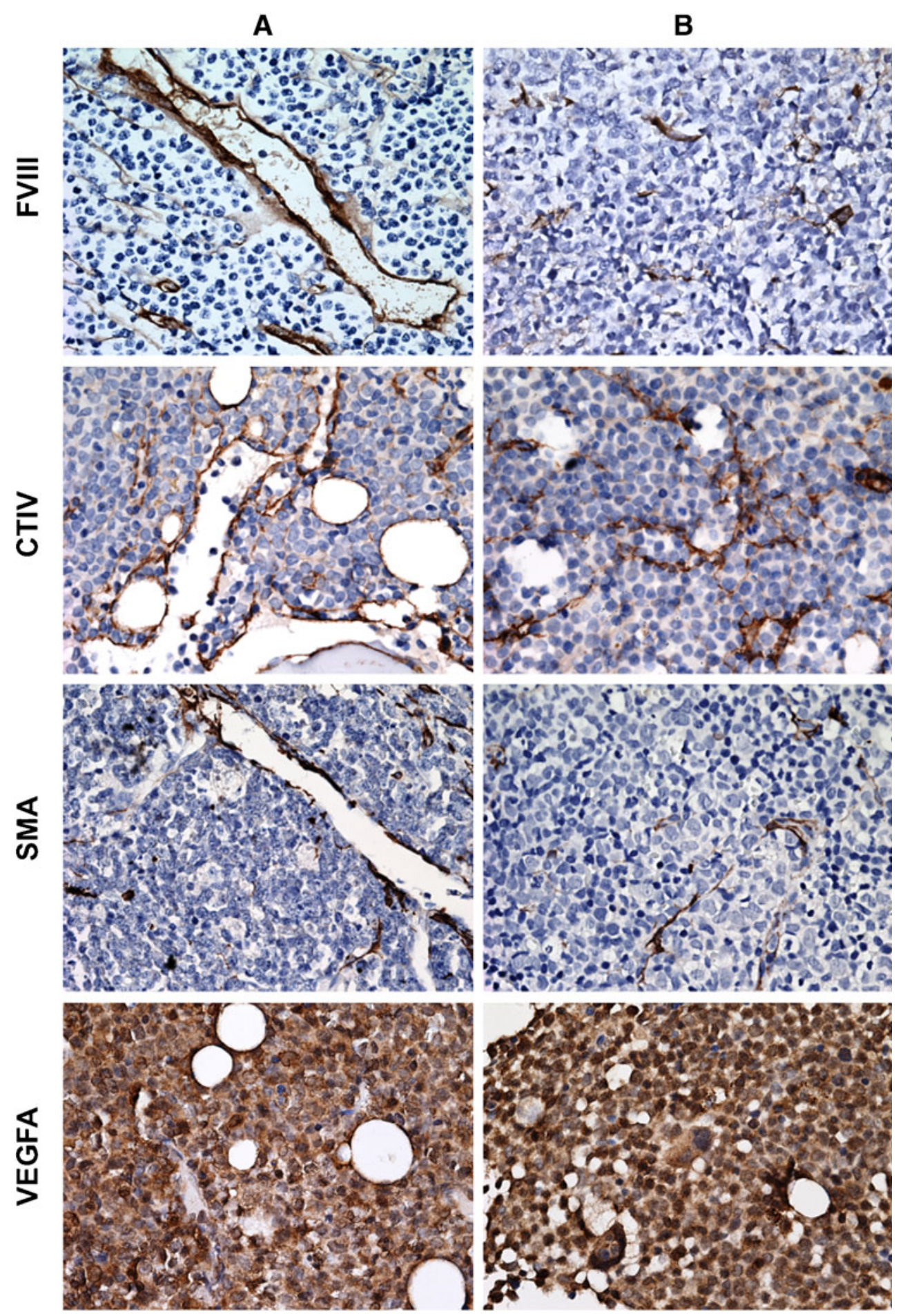

\section{Discussion}

Over the past years it has become clear that new vessel formation is part of the ongoing process in AML; MVD is increased in bone marrow biopsies of patients with newly diagnosed AML compared with normal bone marrow biopsies [5, 21]. In our study we distinguished vessel heterogeneity in AML bone marrow biopsies at diagnosis.
Using vessel count and Chalkley count we were able to divide the AML bone marrow biopsies at diagnosis into three subgroups: a subgroup with a 'low vessel count', a subgroup called 'angiogenic sprouting' (high vessel count, low Chalkley count) and a subgroup 'vessel hyperplasia' (high vessel count, high Chalkley count). To our knowledge, this is the first study that describes different quantified vasculature patterns in AML. 


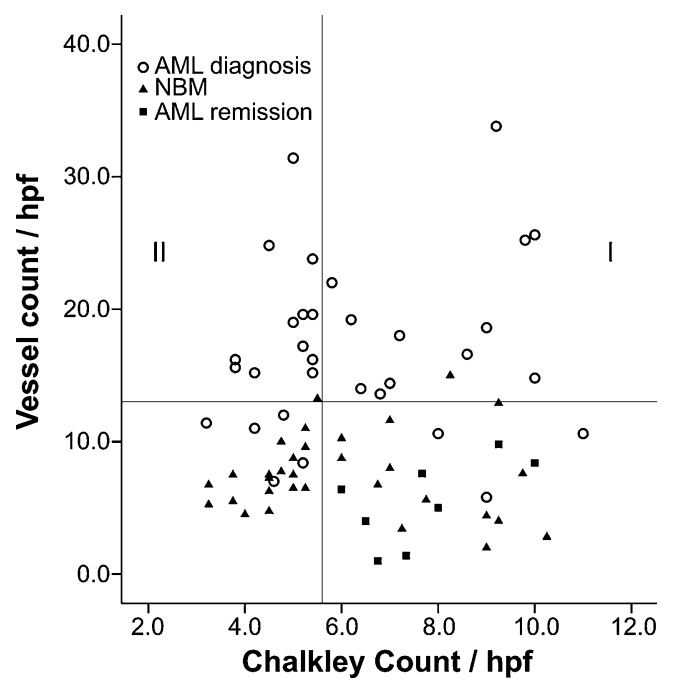

Fig. 3 Scatterplot representing the biopsies of AML at diagnosis, at remission and Normal Bone Marrow. AML biopsies at diagnosis with a 'low vessel count' are displayed below the Y-axis reference line (13 microvessels/hpf), based on the fact that $>95 \%$ of Normal Bone Marrow and AML remission biopsies had a vessel count below 13 microvessels/hpf. The X-axis reference line divides AML biopsies at diagnosis with a 'high vessel count' into two subgroups according to the median Chalkley count of 5.4 in AML at diagnosis and the identical cut-off point set by the median Chalkley count of the NBM and AML remission biopsies. Samples with a Chalkley count $>5.4$ were defined as 'vessel hyperplasia' (group I) and samples $\leq 5.4$ as 'angiogenic sprouting' (group II). AML biopsies: Spearman's rho $0.15, p=0.42$

In solid tumors, the morphology of vessels has been studied extensively. It shows that microvessels are not hierarchically structured and can consist of dilated vessels and/or angiogenic vessel sprouting [2]. These vasculature

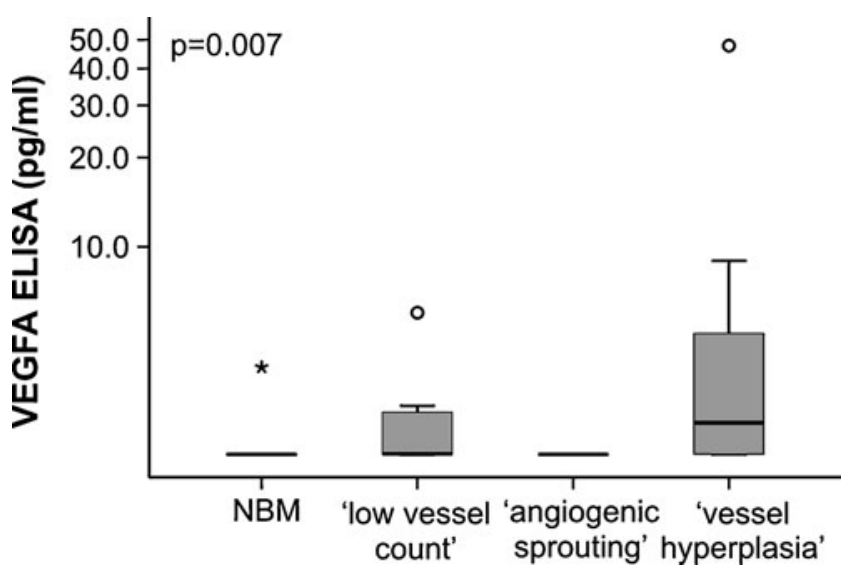

Fig. 4 Relation between AML derived VEGFA protein levels and vessel morphology in AML at diagnosis. The AML-excreted VEGFA protein level was significantly $(p=0.007)$ higher in the subgroup with a 'vessel hyperplasia' morphology compared with the'angiogenic sprouting' or the 'low angiogenic profile'. Box-and-whisker plot limits depict 75 th and 25 th percentiles and median value (box), and upper/lower quantile $\pm 1.5 \times$ (interquantile range) (upper and lower whiskers, respectively) characteristics of solid tumors compromise the delivery and effectiveness of conventional cytotoxic therapies [16]. Treatment with VEGF/VEGFR-signaling interfering drugs induce a 'normalized' vessel pattern as described by vessel remodeling and increased pericyte coverage of tumor vessels $[13,17]$. As a result vessel stabilization and reduced permeability is achieved, facilitating a more efficient delivery of drugs to the targeted cancer cells. In our study we found that a high vessel count in AML bone marrow biopsies is related to a more immature vessel status, implicating that normalization of the vasculature might improve the outcome of chemotherapeutic drugs coadministered.

Drugs targeting VEGF/VEGFR signaling are promising in the treatment of cancer, with an immature vasculature within the tumor being more susceptible. This study shows that AML bone marrow biopsies have a high number of vessels, related to a low percentage of pericyte coverage. Recently, a clinical trial with Bevacizumab, a monoclonal antibody that binds VEGFA, following chemotherapy showed a favorable Complete Remission rate and duration in relapsed and refractory AML [18]. However, heterogeneity in the treatment response was seen during this trial. Since we describe differences in AML vasculature related to VEGFA levels the question raises: do different morphology patterns in AML bone marrow warrant distinct therapeutic approaches, thereby increasing the response? Ongoing work investigating the response of anti-VEGFA therapies related to AML bone marrow vasculature will have to answer this question.

In a xenograft mouse model the appearance of 'vessel hyperplasia' and 'angiogenic sprouting' was demonstrated and seemed to be related to the availability of VEGFA [20]. VEGFA can be released via breakdown of the Extracellular Matrix (ECM). This cleaved variant of VEGFA mainly produces dilated vessels. When VEGFA is not cleaved, and therefore not released from the ECM, it results in an angiogenic sprouting profile [20]. In corroboration with the abovementioned study we showed that biopsies with a high vessel count and a high Chalkley count were related to elevated AML blast derived VEGFA in vitro whereas 'angiogenic sprouting' and 'low vessel count' showed a relative low VEGFA level in vitro. These results support the view that the amount of free VEGFA could play a role in vascular patterning in AML. Interestingly, we also found that patients in the 'vessel hyperplasia' subgroup had a significantly higher platelet count at diagnosis compared with the other two defined subgroups. Since platelets can release VEGFA in vivo, an even higher VEGFA level can be expected enlarging vessel differences even more [19].

In conclusion, our study shows that heterogeneity in AML bone marrow vasculature can be quantified using Chalkley count in addition to vessel count and that biopsies displaying a high vessel count with low pericyte coverage 
could be diverted in two separate entities. Further studies will help to clarify its usefulness for adapting therapeutic approaches in trials with antiangiogenic or VEGFAblocking drugs.

Open Access This article is distributed under the terms of the Creative Commons Attribution Noncommercial License which permits any noncommercial use, distribution, and reproduction in any medium, provided the original author(s) and source are credited.

\section{References}

1. A. Aguayo, E. Estey, H. Kantarjian, T. Mansouri, C. Gidel, M. Keating, F. Giles, Z. Estrov, B. Barlogie, M. Albitar, Cellular vascular endothelial growth factor is a predictor of outcome in patients with acute myeloid leukemia. Blood 94, 3717-3721 (1999)

2. J.W. Baish, R.K. Jain, Fractals and cancer. Cancer Res. 60, 3683 $3688(2000)$

3. J.C. Byrd, K. Mrozek, R.K. Dodge, A.J. Carroll, C.G. Edwards, D.C. Arthur, M.J. Pettenati, S.R. Patil, K.W. Rao, M.S. Watson, P. R. Koduru, J.O. Moore, R.M. Stone, R.J. Mayer, E.J. Feldman, F. R. Davey, C.A. Schiffer, R.A. Larson, C.D. Bloomfield, Pretreatment cytogenetic abnormalities are predictive of induction success, cumulative incidence of relapse, and overall survival in adult patients with de novo acute myeloid leukemia: results from cancer to leukemia group B (CALGB 8461). Blood 100, 4325$4336(2002)$

4. D.J. Crocker, T.M. Murad, J.C. Geer, Role of the pericyte in wound healing. An ultrastructural study. Exp. Mol. Pathol. 13, 51-65 (1970)

5. E.S. de Bont, S. Rosati, S. Jacobs, W.A. Kamps, E. Vellenga, Increased bone marrow vascularization in patients with acute myeloid leukaemia: a possible role for vascular endothelial growth factor. Br. J. Haematol. 113, 296-304 (2001)

6. H.J. de Jonge, E.S. de Bont, P.J. Valk, J.J. Schuringa, M. Kies, C. M. Woolthuis, R. Delwel, N.J. Veeger, E. Vellenga, B. Lowenberg, G. Huls, AML at older age: age-related gene expression profiles reveal a paradoxical down-regulation of p16INK4A mRNA with prognostic significance. Blood 114, 2869-2877 (2009)

7. W.H. Dokter, L. Tuyt, S.J. Sierdsema, M.T. Esselink, E Vellenga, The spontaneous expression of interleukin- $1 \beta$ and interleukin- 6 is associated with spontaneous expression of AP-1 and NF-kB transcription factor in acute myeloblastic leukemia cells. Leukemia (Baltimore) 9, 425-432 (1995)

8. H.F. Dvorak, Vascular permeability factor/vascular endothelial growth factor: a critical cytokine in tumor angiogenesis and a potential target for diagnosis and therapy. J. Clin. Oncol. 20, 4368-4380 (2002)

9. W. Fiedler, R. Mesters, H. Tinnefeld, S. Loges, P. Staib, U. Duhrsen, M. Flasshove, O.G. Ottmann, W. Jung, F. Cavalli, R. Kuse, J. Thomalla, H. Serve, A.M. O'Farrell, M. Jacobs, N.M. Brega, P. Scigalla, D.K. Hossfeld, W.E. Berdel, A phase 2 clinical study of SU5416 in patients with refractory acute myeloid leukemia. Blood 102, 2763-2767 (2003)

10. J. Folkman, Angiogenesis in cancer, vascular, rheumatoid and other disease. Nat. Med. 1, 27-31 (1995)

11. J. Folkman, Angiogenesis and apoptosis. Semin. Cancer Biol. 13, 159-167 (2003)
12. D. Grimwade, H. Walker, G. Harrison, F. Oliver, S. Chatters, C.J. Harrison, K. Wheatley, A.K. Burnett, A.H. Goldstone, The predictive value of hierarchical cytogenetic classification in older adults with acute myeloid leukemia (AML): analysis of 1065 patients entered into the United Kingdom Medical Research Council AML11 trial. Blood 98, 1312-1320 (2001)

13. I. Helfrich, I. Scheffrahn, S. Bartling, J. Weis, V. von Felbert, M. Middleton, M. Kato, S. Ergun, D. Schadendorf, Resistance to antiangiogenic therapy is directed by vascular phenotype, vessel stabilization, and maturation in malignant melanoma. J. Exp. Med. 207, 491-503 (2010)

14. H.A. Hou, T.T. Shih, C.Y. Liu, B.B. Chen, J.L. Tang, M. Yao, S.Y. Huang, W.C. Chou, C.Y. Hsu, H.F. Tien, Changes in magnetic resonance bone marrow angiogenesis on day 7 after induction chemotherapy can predict outcome of acute myeloid leukemia. Haematologica 95, 1420-1424 (2010)

15. T. Inai, M. Mancuso, H. Hashizume, F. Baffert, A. Haskell, P. Baluk, D.D. Hu-Lowe, D.R. Shalinsky, G. Thurston, G.D. Yancopoulos, D.M. McDonald, Inhibition of vascular endothelial growth factor (VEGF) signaling in cancer causes loss of endothelial fenestrations, regression of tumor vessels, and appearance of basement membrane ghosts. Am. J. Pathol. 165, 35-52 (2004)

16. R.K. Jain, The next frontier of molecular medicine: delivery of therapeutics. Nat. Med. 4, 655-657 (1998)

17. R.K. Jain, Normalization of tumor vasculature: an emerging concept in antiangiogenic therapy. Science 307, 58-62 (2005)

18. J.E. Karp, I. Gojo, R. Pili, C.D. Gocke, J. Greer, C. Guo, D. Qian, L. Morris, M. Tidwell, H. Chen, J. Zwiebel, Targeting vascular endothelial growth factor for relapsed and refractory adult acute myelogenous leukemias: therapy with sequential 1-beta-d-arabinofuranosylcytosine, mitoxantrone, and bevacizumab. Clin. Cancer Res. 10, 3577-3585 (2004)

19. C. Kut, F. Mac Gabhann, A.S. Popel, Where is VEGF in the body? A meta-analysis of VEGF distribution in cancer. Br. J. Cancer 97, 978-985 (2007)

20. S. Lee, S.M. Jilani, G.V. Nikolova, D. Carpizo, M.L. IruelaArispe, Processing of VEGF-A by matrix metalloproteinases regulates bioavailability and vascular patterning in tumors. J. Cell Biol. 169, 681-691 (2005)

21. T. Padro, S. Ruiz, R. Bieker, H. Burger, M. Steins, J. Kienast, T. Buchner, W.E. Berdel, R.M. Mesters, Increased angiogenesis in the bone marrow of patients with acute myeloid leukemia. Blood 95, 2637-2644 (2000)

22. M. Sie, E.S. de Bont, F.J. Scherpen, E.W. Hoving, W.F. den Dunnen, Tumour vasculature and angiogenic profile of paediatric pilocytic astrocytoma; is it much different from glioblastoma?, Neuropathol. Appl. Neurobiol. (2010)

23. R.T. Tong, Y. Boucher, S.V. Kozin, F. Winkler, D.J. Hicklin, R.K. Jain, Vascular normalization by vascular endothelial growth factor receptor 2 blockade induces a pressure gradient across the vasculature and improves drug penetration in tumors. Cancer Res. 64, 3731-3736 (2004)

24. P.B. Vermeulen, G. Gasparini, S.B. Fox, C. Colpaert, L.P. Marson, M. Gion, J.A. Belien, R.M. de Waal, E. Van Marck, E. Magnani, N. Weidner, A.L. Harris, L.Y. Dirix, Second international consensus on the methodology and criteria of evaluation of angiogenesis quantification in solid human tumours. Eur. J. Cancer 38, 1564-1579 (2002)

25. F. Yuan, Y. Chen, M. Dellian, N. Safabakhsh, N. Ferrara, R.K. Jain, Time-dependent vascular regression and permeability changes in established human tumor xenografts induced by an anti-vascular endothelial growth factor/vascular permeability factor antibody. Proc. Natl Acad. Sci. USA 93, 14765-14770 (1996) 